\title{
Discrete Hopping Lithiation of ZnO Nanowires
}

A. Kushima, ${ }^{*}$ X. H. Liu, ${ }^{* *}$ G. Zhu, ${ }^{* * *}$ J. Li, ${ }^{*},{ }^{* * *}$ Z. L. Wang, ${ }^{* * * *}$ J. Y. Huang ${ }^{* *}$

* Department of Materials Science and Engineering, University of Pennsylvania, Philadelphia, Pennsylvania 19104

** Center for Integrated Nanotechnologies, Sandia National Laboratories, Albuquerque, New Mexico 87185

*** School of Materials Science and Engineering, Georgia Institute of Technology, Atlanta, Georgia 30332-0245

**** State Key Laboratory for Mechanical Behavior of Materials and Frontier Institute of Science and Technology, Xi'an Jiaotong University, Xi'an, 710049, China

Lithium ion batteries (LIBs) are attracting attention for energy storage device for electric vehicles where high power, energy density, and cyclability are required. Nanowire (NW) electrode [1] has advantage over conventional electrodes due to its unique geometry that enhances the electron and $\mathrm{Li}^{+}$transport. In addition, NWs can accommodate large volume change during charge/discharge cycles [2], leading to the improved cyclability and stability. In this study, the lithiation processes of individual $\mathrm{ZnO} \mathrm{NW}$ electrodes in a LIB configuration were observed by in-situ transmission electron microscopy (TEM) using a unique nano-battery setup inside the TEM [3] developed recently for observing the electrochemistry processes in real time.

The nano-battery consisted of a single $\mathrm{ZnO} \mathrm{NW}$ as an anode, an ionic-liquid electrolyte (ILE), and $\mathrm{LiCoO}_{2}$ particles as cathode. Figure 1 shows the lithiation process of the $\mathrm{ZnO}$ NW. The initial NW was straight and had smooth surface (Fig. 1A). After contact with ILE, a potential of $-4.0 \mathrm{~V}$ with respect to the $\mathrm{LiCoO}_{2}$ counter electrode was applied to the $\mathrm{ZnO}$ NW (Fig.1B). The solid-state reaction front propagated along the longitudinal direction of the NW away from the ILE (Fig. 1B-K). As the reaction front propagated, the diameter and the length of the NW increased, causing the NW to bend. Figure 1L-O shows detailed view of the reaction front propagation. Interestingly, the reaction front did not move progressively along the NW. Instead, it advanced by initiating discrete cracks about $80 \mathrm{~nm}$ before the reaction front (Fig. 1M, red arrowheads). The lithiation then propagated laterally along its two side (Fig. $1 \mathrm{~N}-\mathrm{O}$ ). The cracks divided the NW into smaller segments. The new crack grew in a similar fashion to the old crack, and this process repeated until the entire nanowire was lithiated. From the above observations, the lithiation of the $\mathrm{ZnO} \mathrm{NW}$ consists of three steps. 1) The $\mathrm{Li}^{+}$adsorbs on the NW surface initiating the lithiation. 2) The reaction leads to crack formation in the NW making path from the surface inward the bulk. 3) $\mathrm{Li}^{+}$penetrates into the NW from the crack and reacts with the NW. The lithiation process is schematically illustrated in Fig. 2A. The crack formation during the lithiation process caused the $\mathrm{ZnO}$ NW to break into multiple segments (Fig. 2B).

The fracture of the NW is considered to cause poor cyclability of the battery when $\mathrm{ZnO}$ is used as the LIB electrode. Our observations provide important insight for developing battery with higher performance and longer lifetime by providing the failure mechanism of the electrode material [4]. 
References

[1] C. K. Chan et al., Nat. Nano. 3 (2008) 31.

[2] A. Magasinski et al., Nat. Mater. 9 (2010) 353.

[3] J. Y. Huang et al., Science 330 (2010) 1515.

[4] This research was supported by Honda Research Institute USA, NSF grants DMR-1008104 and DMR-0520020, and Air Force Office of Scientific Research grant FA9550-08-1-0325. Sandia National Laboratories is a multi-program laboratory operated by Sandia Corporation, a wholly owned subsidiary of Lockheed Martin Company, for the U.S. Department of Energy's National Nuclear Security Administration under contract DE-AC04-94AL85000.
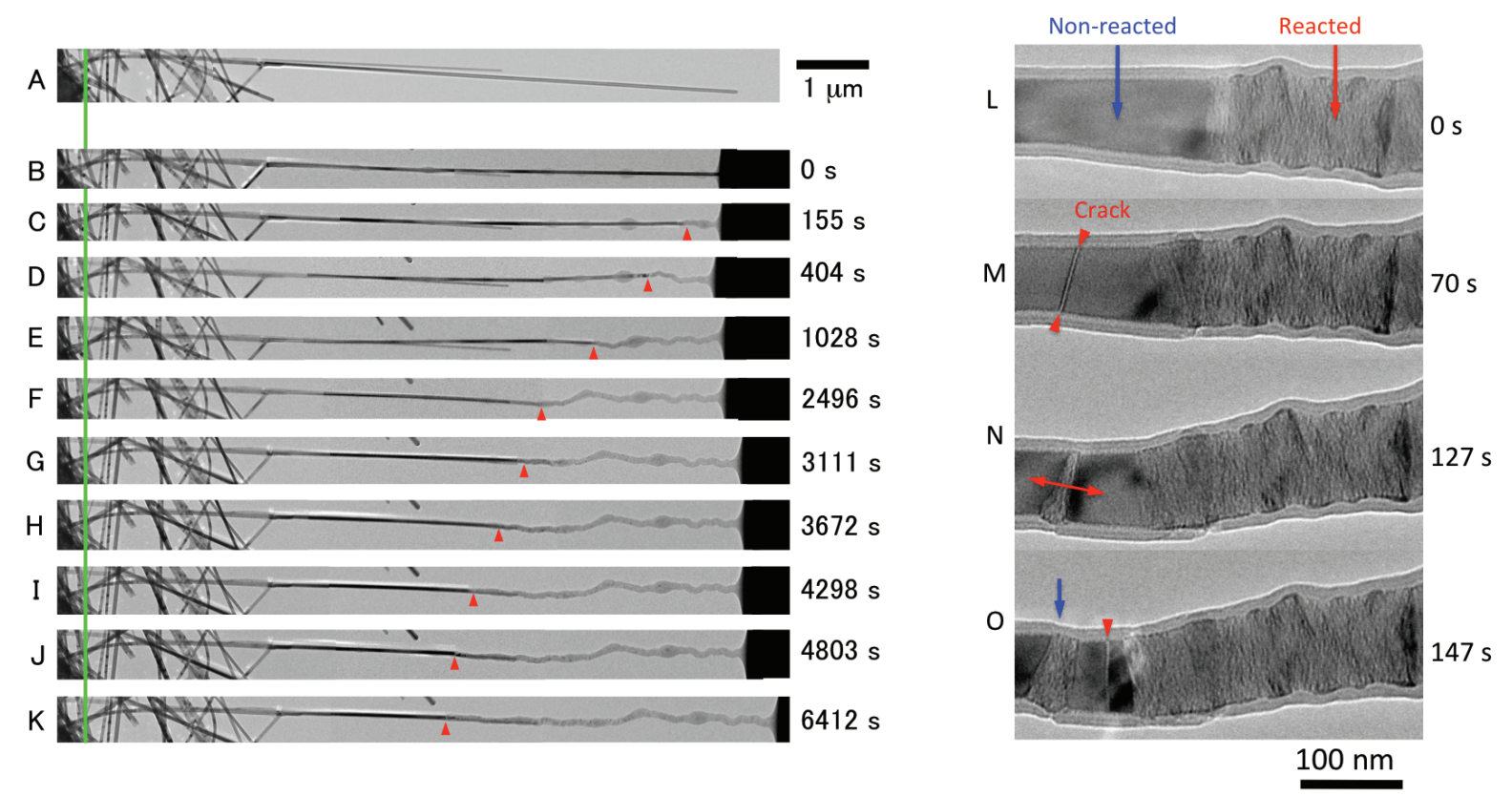

FIG. 1. (A-K) Typical lithiation process of the $\mathrm{ZnO}$ nanowire. Triangles in the figure indicate the propagation of the reaction front during the lithiation. (L-O) Discrete crack nucleation ahead of the reaction front. Cracks formed near the reaction front in the non-reacted part of the $\mathrm{ZnO} \mathrm{NW}$ as indicated by the triangles in the figure separating the NW into multiple segments.
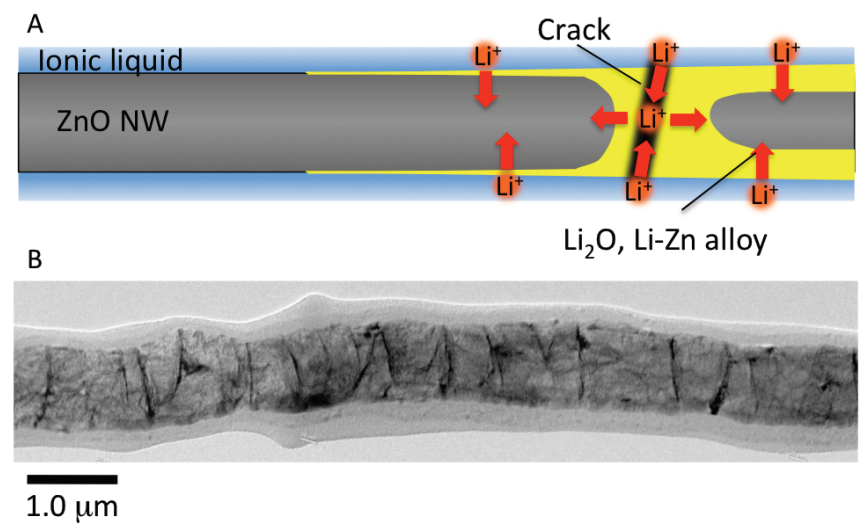

FIG. 2. (A) Schematic illustration explaining the lithiation mechanism of $\mathrm{ZnO} \mathrm{NW}$. The lithiation first takes place at the surface of the NW forming a crack from the surface inward the core. Then, the $\mathrm{Li}^{+}$penetrates inside the NW from the crack. As a result, the NW was separated into multiple segments after the lithiation (B). 